\title{
Absolute quantification of stool-based colon cancer microRNAs by chip-based digital (d)PCR
}

\section{Farid E Ahmed* \\ GEM Tox Labs, Institute for Research in Biotechnology, USA}

The expression of individual genes may be altered by mutations in the DNA, or by a change in their regulation at the RNA or protein levels. Epigenetic silencing is an important mechanism that contributes to gene inactivation in colorectal cancer (CRC). Analysis of promoter methylation of hypermethylated in cancer 1 (HIC1) gene in stool showed it to be highly specific ( $98 \%$ ) for colon adenoma and carcinoma, but sensitivity was quite low ( $31 \%$ for adenoma $\& 42 \%$ for all cancer), which suggested that an epigenetic marker only is not adequate for screening, but a combination of genetic and epigenetic markers would be required to reliably identify CRC at an early stage.

Working with the stable DNA has been relatively easy. A study by scientists affiliated with Exact Sciences Corp., Marlborough, MA, which markets a mutation-based DNA test, assessed a newer version of a fecal DNA test for CRC screening using a vimentin methylation marker and another mutation DY marker plus non degraded DNA in a limited sample of 44 CRC patients and 122 normal controls. It cited a sensitivity of $88 \%$ and a specificity of $82 \%$ only for advanced cancer, but not adenoma. Besides, DNA mutation tests are not cost-effective, as screening for multiple mutations is expensive because these demanding mutation tests are not automated and are labor intensive. In addition, mutation detection in oncogenes and suppressor genes suffers from: a) the detection of mutations in these genes in fewer than half of large adenomas and carcinomas, b) the detection of gene mutations in nonneoplastic tissues, c) mutations found only in a portion of the tumor, and d) mutations often produce changes in the expression of many other genes.

Protein-based methods are currently not suited for screening and early diagnosis either because proteins are not specific to one tumor or tissue type (e.g., CEA), their susceptibility to proteases, current lack of means to amplify proteins, no function is known for more than $75 \%$ of predicted proteins of multicellular organisms, there is not always a direct correlation between protein abundance and activity, and most importantly because detection of these markers exfoliately often signifies the presence of an advanced tumor stage. The dynamic range of protein expression in minimally-invasive body fluids (e.g., blood) is as large as $10^{10}$. Moreover, mRNA levels do not necessarily correlate with protein expressions. Protein microarray studies revealed that protein expression vastly exceeds RNA levels, and only posttranslationally modified proteins are involved in signal transduction pathways leading to tumorigenesis. There is no well-documented protein test that has been shown in clinical trials to be a sensitive and a specific indicator of colon neoplasia, especially in early stages. More recently, a serum proteomic study employing liquid chromatography (LC)mass spectrometry (MS) carried out in a nonbiased fashion failed to differentiate between individuals with large adenoma $(\leq 1 \mathrm{~cm})$ and normal individuals. Proteomic research is a relatively new discipline, so it will take considerable time to identify and validate proteins suitable for use as clinical markers, and resolve issues of bias and validations.

On the other hand, a transcriptomic mRNA approach has shown promise to detect adenomas and colon carcinomas with high sensitivity and specificity in preliminary studies, but no randomized, standardized, blinded prospective clinical study has been carried out to validate the superiority of the mRNA approach.

The discovery of small noncoding protein sequences, 1727 nucleotides long stable RNAs [micro(mi)RNAs], has opened opportunities for developing a non-invasive screening test for early diagnosis of many cancers. MiRNA functions seem to regulate development, apoptosis, and specific miRNAs are essential in oncogenesis, effective in classifying solid and liquid tumors, and serve as oncogenes or suppressor genes. MiRNA genes are frequently found at fragile sites, and minimal regions of loss of heterozygosity, or amplification of common break-point regions, implying their involvement in carcinogenesis; and they have potential to serve as biomarkers for cancer diagnosis, prognosis and/or response to therapy. Profiles of miRNA expression differ between normal and tumor tissues, suggesting that their expression profiles cluster similar tumor types together more accurately than expression profiles of protein-coding messenger(m)RNA genes. Unlike screening for large numbers of mRNAs, a modest number of miRNAs is used to differentiate cancer from normal, and unlike mRNA, miRNAs in stool remain largely intact and stable for detection. Therefore, miRNAs are better molecules to use for developing a reliable noninvasive diagnostic screen for colon cancer. Innovation of the dPCR-miRNA stool screening approach lies in the collective use of many methods, such as: immunoparamagnetic beads to capture colonocytes from the harsh, noninvasive stool environment, whose extracted fragile total small RNA is stabilized shortly after stool excretion by commercial kits so it does not ever fragment, followed by standardized analytical quantitative miRNA dPCR-chip profiling in stool samples, which are neither labor intensive, nor require extensive sample preparation, to develop a panel of few stable miRNAs for absolute quantitative diagnostic screening of early sporadic colon cancer (stage $0-1)$, cheaper, with higher sensitivity and specificity than any other colon cancer screening test on the market. Unlike screening for large numbers of messenger ( $\mathrm{m}$ )RNA, a modest number of miRNAs can be

*Correspondence to: Farid E Ahmed, GEM Tox Labs, Institute for Research in Biotechnology, 2905 South Memorial Drive, Greenville, NC 27834, USA, Tel: (252) 689-5681, E-mail: gemtoxconsultants@yahoo.com

Received: March 15, 2019; Accepted: March 28, 2019; Published: April 01, 2019 
used to differentiate cancer from non-cancer condition, and unlike mRNA, miRNAs in stool remain largely intact and stable for detection. Therefore, miRNAs are better molecules to use for developing a reliable noninvasive diagnostic screen for colon cancer, since: a) the presence of the bacterium Escherichia coli in stool does not hinder detection of miRNA by a sensitive technique such as dPCR, and b) the miRNA expression patterns are the same in primary tumor, or diseased tissue, as in stool samples.

Copyright: $\odot 2019$ Ahmed FE. This is an open-access article distributed under the terms of the Creative Commons Attribution License, which permits unrestricted use, distribution, and reproduction in any medium, provided the original author and source are credited. 